\title{
A Proactive Ant Colony Algorithm for Efficient Power Routing using MANET
}

\author{
N.Umapathi \\ Research Scholars \\ Electronics and \\ Communication Engineering, \\ GKM college of Engg and \\ Tech, \\ Chennai-63, India.
}

\author{
N.Ramaraj, PhD. \\ Professor \\ Dept. of Computer Science \\ Engineering, \\ GKM college of Engg and \\ Tech, \\ Chennai-63, India.
}

\author{
R.Adlin Mano \\ M.E Communication System, \\ Dept.Electronics and \\ Communication Engineering \\ GKM College of Engg and Tech, \\ Chennai-63, India.
}

\begin{abstract}
The field of wireless networks is an important and challenging area. In this paper we describe, AntHocNet an algorithm for routing in mobile adhoc networks. It is a hybrid algorithm which combines proactive and reactive behavior to compute packet delivery ratio, end to end delay and overhead by varying the speed of the mobile nodes. The algorithm is based on proposed nature inspired, self organized algorithm of ANT colony optimization (ACO). The bit error rate of ANT algorithm in accordance with other algorithms (AODV, DSDV, DSR, TORA......) is computed including power consumption, time delay and packet loss.
\end{abstract}

KEYWORDS- ANT Colony Optimization, Wireless network, Mobile Adhoc Network (MANET), AntHocNet.

\section{INTRODUCTION}

Mobile Adhoc Networks (MANETS) is a group of mobile nodes which communicates with each other without any supporting infrastructure. The need for MANETS is growing and it can be connected to wired or wireless links using one or more different technologies. MANETS should be self built, self configured and adaptive to dynamic changes. In this paper, the application of ANT routing algorithm for mobile adhoc networks is introduced. Here we concentrate on power consumption in the nodes. ANT colony optimization (ACO) method is used for optimum route discovery in wireless networks. It is a class of constructive algorithms, the cooperative behavior of real ants to different optimization problems.

\section{RELATED WORK}

\section{A. Overview of Ant algorithm}

ANTNET and ANTHOCNET are two well known ANT colony based routing algorithms. AntHocNet has a very high delivery rate and find routes to find the length of the shortest path. Here POSANT (position based ANT colony routing algorithm) is being implemented to control the number of control messages which makes the routing scalable. The application of this proposed algorithm is it utilizes remaining power to forward the neighbor node. The remaining power helps the source or intermediate node to distribute the power for forwarding in order to avoid routing holes problem. This algorithm cannot be directly applied to MANETS.

\section{B. Overview of AntHocNet algorithm}

In this paper, AntHocNet design is based on self organizing behavior of Ant, ANT colony optimization and shortest path delivery. AntHocNet follows a hybrid approach ants are generated in relation to both proactive and reactive schemes. AntHocNet has a performance advantage over AODV. The advantage exists in metrics like packet delivery ratio, average end to end delay and average jitter in a more dynamic environment.

\section{LITERATURE SURVEY}

\begin{abstract}
A.MANETS
Adhoc networks are classified based on their applications in to mobile adhoc networks (MANETS), wireless mesh networks (WMN), wireless sensor networks (WSN). MANETS are very popular in different applications considering the following factors: reliability, cost, bandwidth, and total required power, ease of installation, security and performance of network. Manet has several salient features: resource constraints, dynamic topology, no infrastructure and limited physical security. MANET is an infrastructure less network since the mobile nodes in the network establish routing to form their own network. The topology is highly dynamic. The time between fault detection and restoration of new valid path is referred to as convergence time. It reflects the ability of the algorithm to the challenging network dynamics. Due to increase in complexity and risks associated with operation and management it is important in mobile adhoc networks.
\end{abstract}

The resulting increase in complexity and potential risks associated with operation and management. It is becoming important that mobile adhoc networks have mobile adhoc networks have certain self organization properties ranging from self configuration during the start up to self adaptation to changes in operating environment.

\section{B.ANTHOCNET}

In anthocnet we aim to design an algorithm which works efficiently in Manets. The design is based on self organizing behavior of ants. It is a dynamic topology. When network topology changes, it must be restored quickly and this is achieved through a new route delivery process. Information can be enhanced when the resources are being accelerated. Due to this the network may collapse so if convergence time is increased, then network is not being overloaded. If we decrease convergence time, then the network is overloaded. AntHocNet emerges as a hybrid algorithm as it combines reactive, proactive, adaptive, and multipath algorithm and set up the 
route to destinations. It setup the paths and does not maintain the path to destination. In reactive path setup phase, the reactive agents are called as forward ants which are done by the source in order to find the multiple paths to the destination and the backward ants return to setup the paths. These paths are represented in pheromone tables indicating their quality. After the path is setup data packets are routed as datagrams over the path used in this pheromone tables.

It is a proactive agent collecting information and it discovers new routes which act as alternatives if a link fails. This is a multipath because because it provides different routes to send information to the destination.

\section{Real ants mechanism}

The basic idea of ANT colony optimization is taken from the food searching behavior of real ants. When ant searches for food, they start from nest and walk towards the food. When ant reaches an intersection, ant has to decide which branch to take next while walking. Ants deposit a substance called pheromone. This marks the route taken. The concentration of pheromone decreases due to diffusion effects. The behavior of ants is used to find the shortest route in the networks.

\section{FLOWCHART FOR WORKING ANTHOCNET}

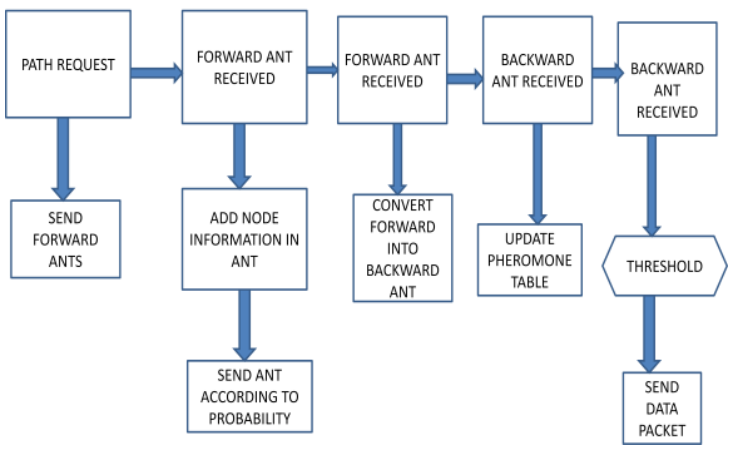

Fig1.1Working AntHocNet

\section{Ant colony optimization suits to ADHOC networks}

The important properties of mobile adhoc networks are ACO is based on agent systems and works with individual ants.

- ACO is based on local information. Here no routing tables or information blocks have to be transmitted to all the nodes of the network.

- It is possible to integrate link quality for computation of pheromone concentration.

- Each node has a routing table with entries of its neighbors which contains the pheromone concentration.

\section{SYSTEM DESIGN AND IMPLEMENTATION.}

\section{a. Design criteria}

Simulator chosen to evaluate the protocols is NS2. It has important advantages when compared to other simulators. Number of nodes can exceed several thousands. It has more efficient routing tables. It is a binded model between $\mathrm{c}++$ and OTCL. Wireless network performance mainly depends upon end to end throughput and average delay. It is cost effective of network deployment as wiring is not possible.

\section{b. Algorithms chosen}

A Bioinspired algorithm chosen for comparison of some kinds of algorithm like AntHocNet, AODV, DSR, DSDV, TORA... These algorithms are the principles of the type of algorithm they represent.

\section{c. Implementation}

NETWORK SCENARIOS: In order to evaluate the performance of the proposed protocol we have used network simulator NS-2.32 and have the modifications of AODV. The improvement of AODV depends on four cases:

- Number of stationary modes which are running the same MANET routing protocol with default parameters but here simulation of time is configured.

- $\quad$ FTP is configured to generate high traffic loads and concurrent connections repeats during the whole simulation

- Mobile nodes positioned randomly in area which are running the same MANET protocol.

- Once the node reaches the destination, another new random destination is targeted starting from the previous random destination after a pause time.

Evaluation measures: We distinguish between measures of efficiency and measures of effectiveness. Effectiveness measures the performance. We use four different measures of effectiveness.

- The first one is packet delivery ratio. It is the ratio of delivered data packets versus sent packets.

- This is the cumulative statistical measure of the delays experienced by the packets travelling between source and destination.

- Average jitter is the third measure which is the variation in time interval between the arrivals of subsequent packets.

- Atlas throughput is the measure of number of packets sent in unit time.

We consider two different measures of efficiency.

- The first is overhead in number of packets. It is the total number of control packets transmitted by the nodes in the network.

- The second is average queuing delay which is the average amount of time the packets spends waiting to be dequeued.

\section{TESTING}

The node mobility and node density is being varied in steps to construct a network topology graph which is described randomly. For the bio-inspired routing algorithm the program is written in $\mathrm{C}++$ programming language. Each link is bidirectional and the weighting value of the link depends on the power consumption and ant's moving time delay. After the source node produces a quantity of artificial ants or packets conforming to the Poisson distribution, the destination nodes are randomly chosen with the average probability. A fixed size of one packet is considered in this simulation. 


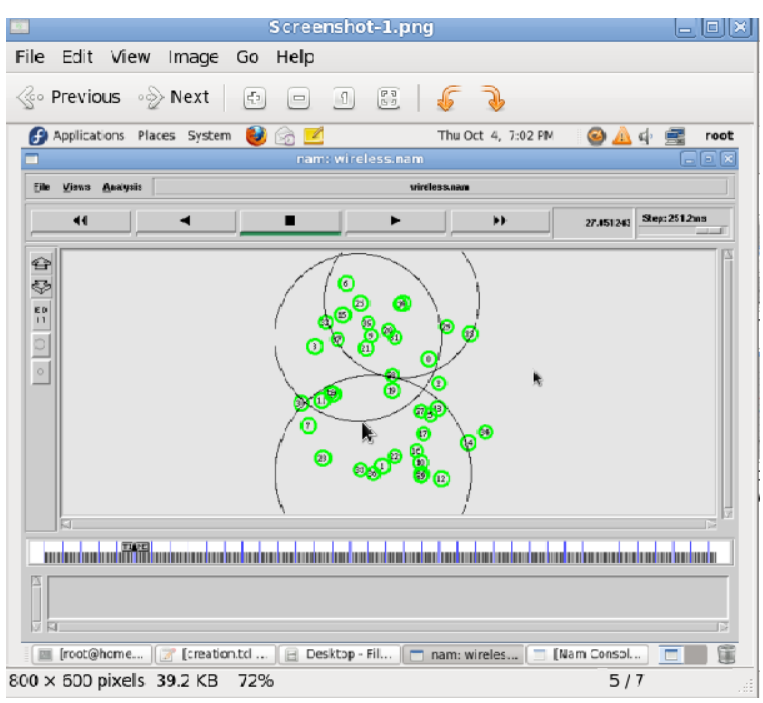

Fig1.2 Network Topology using fedora software.

The experimental parameters are used to configure the system.

\section{Average Jitter}

Jitter improves with increasing datarates and this is due to the fact that data packets are send more frequently,with subsequent packets travel more close to each other and more similar condition the delay is smaller.

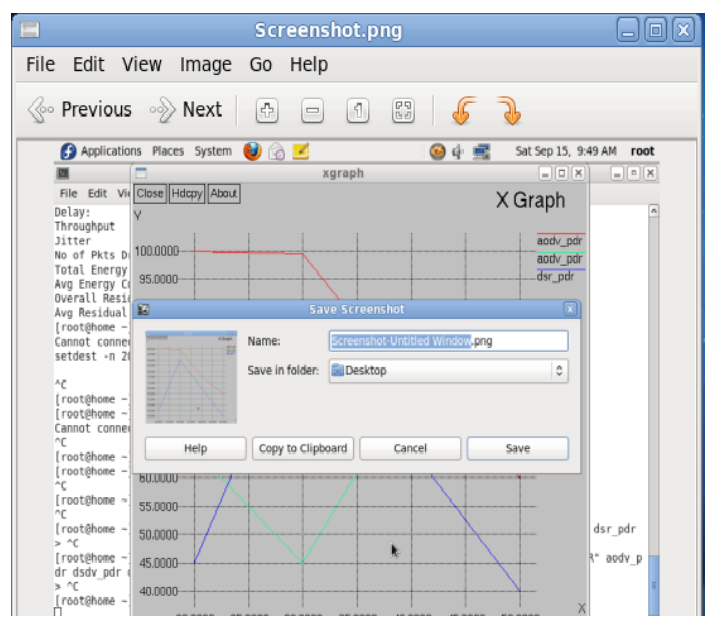

Fig 1.3 Average Jitter versus throughput.

\section{Average end to end delay}

The tests conducted on average end to end delay with varying the number of nodes. It can be observed that AntHocNet has lower end to end delay when compared to AODV. At high pause time end to end delay increases. This is because nodes are concentrated more in the centre of the network rather at the edges for low pause times.

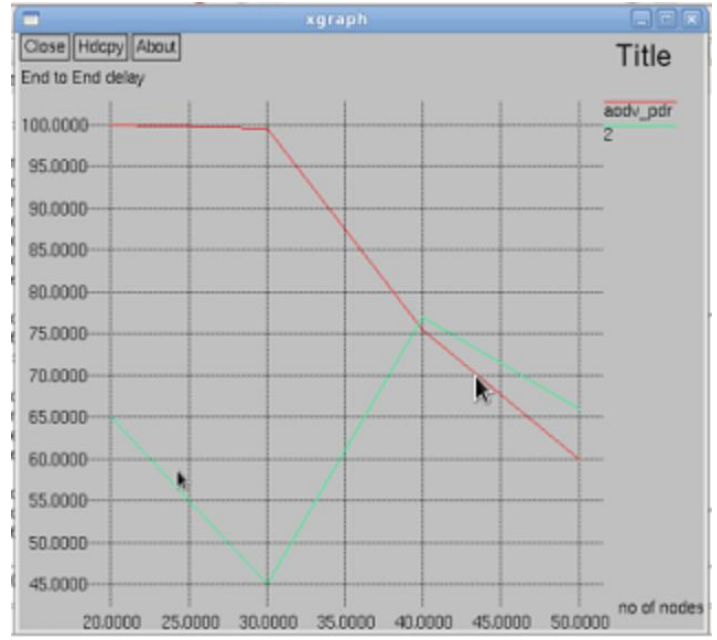

Fig1.4 Average packet delay versus Network size.

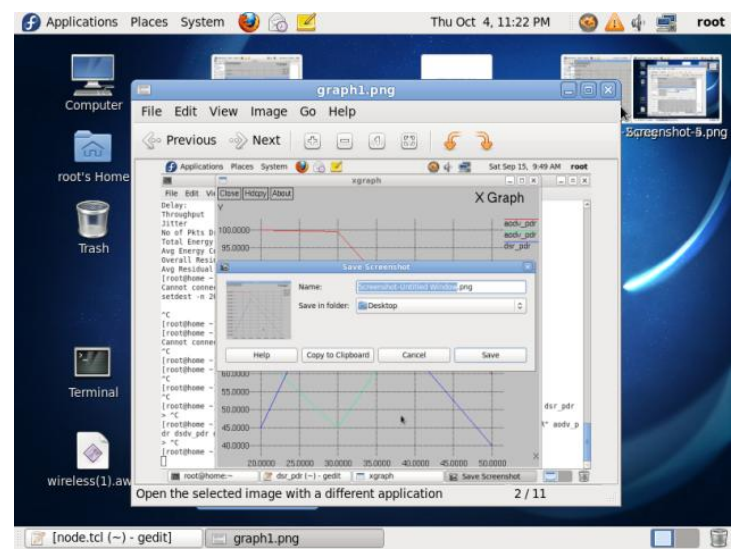

Fig1.5 Delivery ratio under increasing network size.

\section{C.SIMULATION}

The performance gain by optimization algorithm becomes more significant. The proposed protocol can deliver more packets to the destination in shorter time and with low overhead than other protocols. The packet delivery ratio, end to end delay, overhead, throughput jitter energy consumed, normalized routing overheads and average residual energy consumed can be observed. However, results are similar to those presented in this paper. Hence data sent along these routes decreases, reducing the overall throughput.

$\begin{array}{ll}\text { No of pkts send } & 977 \\ \text { No of pkts recv } & 897 \\ \text { Pkt delivery ratio } & 91.8117 \\ \text { Control overhead: } & 981 \\ \text { Normalized routing overheads } & 1.09365 \\ \text { Delay: } & 0.0119797 \\ \text { Throughput } & 95495.8 \\ \text { Jitter } & 0.0410005 \\ \text { No of Pkts Dropped } & 80 \\ \text { Total Energy Consumption: } & 148.786 \\ \text { Avg Energy Consumption: } & 3.81504 \\ \text { Overall Residual Energy: } & 241.198 \\ \text { Avg Residual Energy: } & 6.18457\end{array}$

Fig1.6 Simulation Results 


\section{CONCLUSION}

In this paper we have proposed an algorithm, where the ant agents finds a path when pheromone left before by other ant agents, it does not broadcast itself instead it takes the path of the previous ant agent and leaves more pheromone as it returns back. The comparison is based on the performance metrics, packet delivery ratio, average jitter, throughput, queuing delay, control packet overhead and average end to end delay. NS2 is chosen to simulate this algorithms and a $\mathrm{C}++$ script is written to measure the metrics.

\section{FUTURE ENHANCEMENTS}

For further improvements, pheromone diffusion can be done in order to improve the behavior of proactive ants. This is mainly to limit the routing information. The routing information will be too unreliable. The extra information is used to regulate the generation rate of proactive ants the improvement of the ant behavior helps to reduce the overhead created by the algorithm ants generate in an intelligent and adaptive way to provide results at lower cost. Finally this routing mechanism will come up with better success rate, time and energy consumption.

\section{REFERENCES}

[1] H.Yang, H.Luo, F.Ye,S.Lu, and L. Zhang, Security in Mobile Adhoc Networks IEEE Wireless Communications, pp. 38-47, 2004.

[2] H. Wedde, M. Farooq, T. Pannenbaecker, B. Vogel, C. Mueller, J. Meth and R. Jeruschkat : "BeeAdHoc : an energy efficient routing algorithm for mobile adhoc networks inspired by bee behavior", Proceeding of the Genetic and evolutionary Computation Conference (GECCO) ,Washington DC, USA, pp. 153-160, June (2005).

[3] S.Rajagopalan and C.Shen:"ANSI: a swarm intelligence based unicast routing protocol for hybrid ad hoc networks", Journal of System Architecture, 52, 8-9, pp.485-504 (2006).

[4] Goss S, Aron S, Deneubourg JL, Pasteels JM. Selforganized shortcuts in the Argentine ant Naturwissenschaften 1989; 76:579-581.
[5] Theraulaz G, Bonabeau E. A brief history of stigmergy. Artificial Life, Special Issue on Stigmergy, 1999; 5:97116.

[6] Fewell JH. Social insect networks. Science 2003; 301(26):1867-1870.

[7] Camazine S, Deneubourg J-L, Franks NR, Sneyd J, Theraulaz G,Bonabeau E. Self-Organization in Biological Systems. Princeton University Press: Princeton, NJ, 2001

[8] Cete ME, Doyle JC. Reverse engineering of biological complexity. Science 2002; 295(1):1664-1669.

[9] Abolhasan M, Wysocki T, Dutkiewicz E. A review of routing protocols for mobile ad hoc networks. Adhoc Networks 2004; 2:1-2:2

[10] Gianni Di Caro, Frederick Ducatelle and Luca Maria Gambardella European Transactions on Telecommunications, 2005; 16:443-455: DOI: 10 , 1002/ett.1062

[11] Nilesh P. Bobade ,Nitiket N. Mhala ,Performance Evaluation of Adhoc on Demand Distance Vector in MANETS with varying Network Size using NS2 Simulation. Internation Journal on Computer Science and Engineering: Vol.02, No.08, 2010, 2731-2735.

[12] Ravinder Ahuja, Simulation based Performance Evaluation and Comparison of Reactive, Proactive and Hybrid Routing Protocols based on Random Waypoint Mobility Model, International Journal of computer Applications (0975 - 8887) Volume 7- No.11, october2010 International Journal of Computer Applications (0975 -8887)Volume 7- No.11, October 2010.

[13] Fernando Correia, Teresa Vazao M. Young, The Technical Writer's Handbook. Mill Valley, CA: University Science, 1989.

[14] Annapurna P Patil, Narmada Sambaturu, Krittaya Chunhaviriyakul Convergence Time Evaluation of Algorithms in MANETs. International Journal on Computer Science and Information Security: Vol.XXX, No.XXX, 2009. 